\title{
Chromosome Structure in the Course of Mitosis
}

\author{
G. Giménez-Martín and López-Sáez, J.F. \\ Institudo de Biología Celular (C.S.I.C.), Sección de Citología, \\ Velazquez, 138, Madrid (6), Spain
}

Received May 15, 1964

The chromosome has become one of the most investigated structures of the cell; but despite this, the pattern of somatic chromosome coils, the degree of subdivision as it is observed with the light microscope, the structure of the chromosome at its different levels and the origin of the spiralization, as well as the "spiralization and despiralization cycle", are today matters of controversy.

In two preceeding communications, we have published our findings on the pattern of structure observed in the chromosomes of Scilla non-scripta. A new series of observations have induced us to undertake a wider survey of the questions mentioned above.

\section{Material and methods}

Root-tips of Scilla non-scripta were studied with or without 8oxyquinolein pre-treatment. Tjio and Levan's staining technique (1950) was employed.

\section{Observations}

Number of strands In the prophase two chromatids can be observed in each chromosome and two sub-chromatids forming each chromatid.

In the metaphase, the sub-chromatid was still more clearly observed all along each chromatid. The chromosomes appear as made up of four strands, forming a plectonemic coil two by two. Thus, two sub-chromatids (halfchromatids) make up a chromatid and two chromatids a chromosome. At certain points in some sub-chromatids a double structure could be observed.

Anaphase When the chromatids migrate to the poles, their structure shows two subchromatids, coiled about each other, forming a plectonemic coil. This structural pattern is observed all along the chromatid, and not only at the ends. The structure of the sub-chromatid was more clearly observed than in the metaphase, and shows two half-sub-chromatids plectonemically coiled. In this way, the subchromatid appears also to be formed by two filaments (half-sub-chromatids).

Telophase At the free ends of some chromatids an eightfold structure could be observed, showing eight chromomere. However, in these cases no 
filament was observed, and therefore it is not possible to affirm a double structure for the half-sub-chromatid.

In short: Each chromosome shows two chromatids; each chromatid two sub-chromatids, and each sub-chromatid two half-sub-chromatids. The prophase chromosome appears to be formed by eight strands (half-subchromatids), associated two by two to form the sub-chromatid level, the sub-chromatids two by two make up the chromatids, and these, two by two, the chromosome.

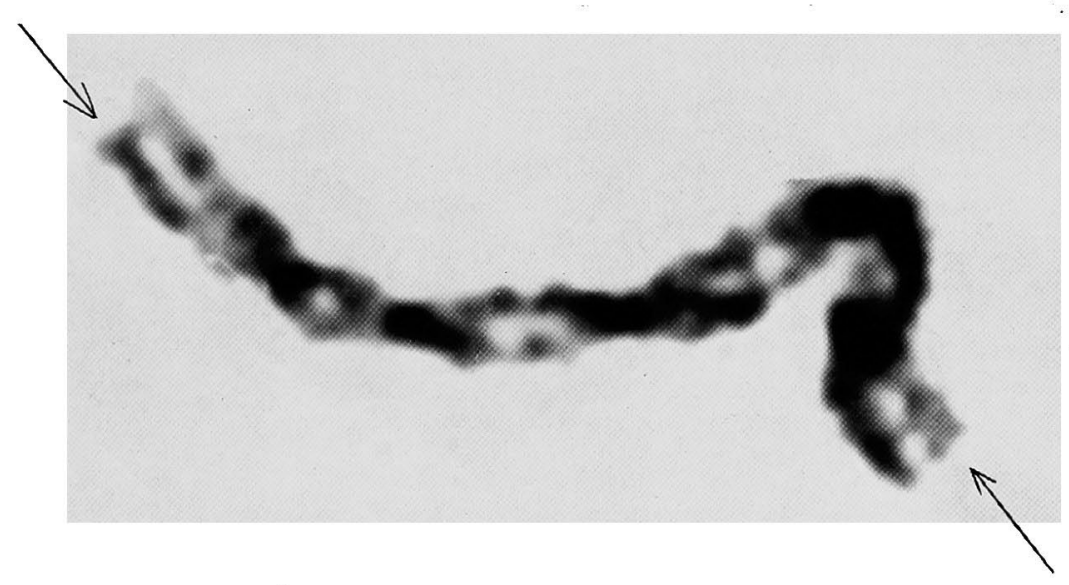

a

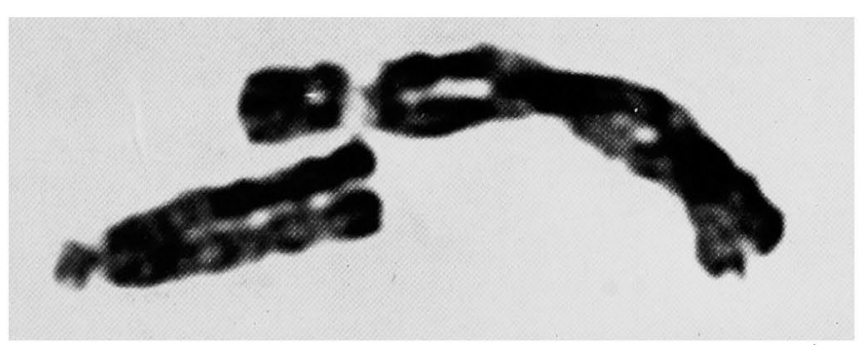

b

Fig. 1. a, plectonemic coil between the chromatids of a prometaphase chromosome. At the free ends the double structure of the chromatids can be observed. b, in two metaphase chromosomes, the plectonemic coil between chromatids and between sub-chromatids is shown.

Pattern of coiling The levels of chromatids, sub-chromatids and halfsub-chromatids apparently present the same pattern of coiling. The chromatids are interwined to form a chromosome: the sub-chromatids are also plectonemically coiled about each other and, in the same way, the half-sub-chromatids make up a plectonemic coil. The pattern of coiling at each level agrees with that of the plectonemic coil described by Sparrow (1941), the unbalanced spiral by Abraham (1939) the twisted spiral by Manton (1939), the orthospiral by 
Kuwada (1939) and the relational coil by Darlington. The three levels show the same pattern of coiling and there is no room for doubt as to the plectonemic pattern.

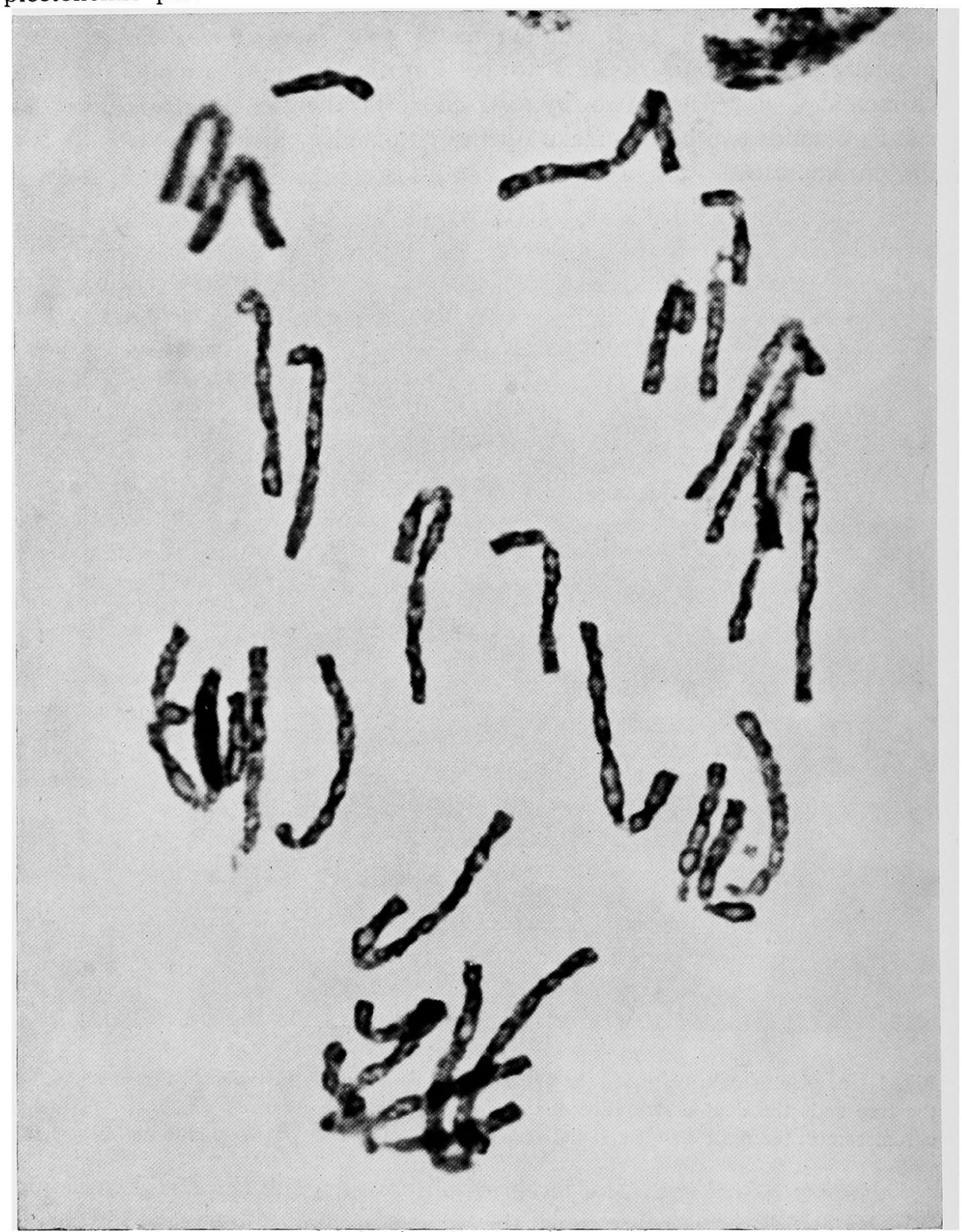

Fig. 2. Anaphase chromosomes showing the plectonemic coil between the sub-chromatids of each chromatid (without any treatment).

\section{Discussion}

Light microscope studies of chromosome structure The chromosome appears today as a complex aggregate of basic 
structural units, and it is generally believed that the minimum number of strands of an anaphasic chromosome is two. On the other hand, several

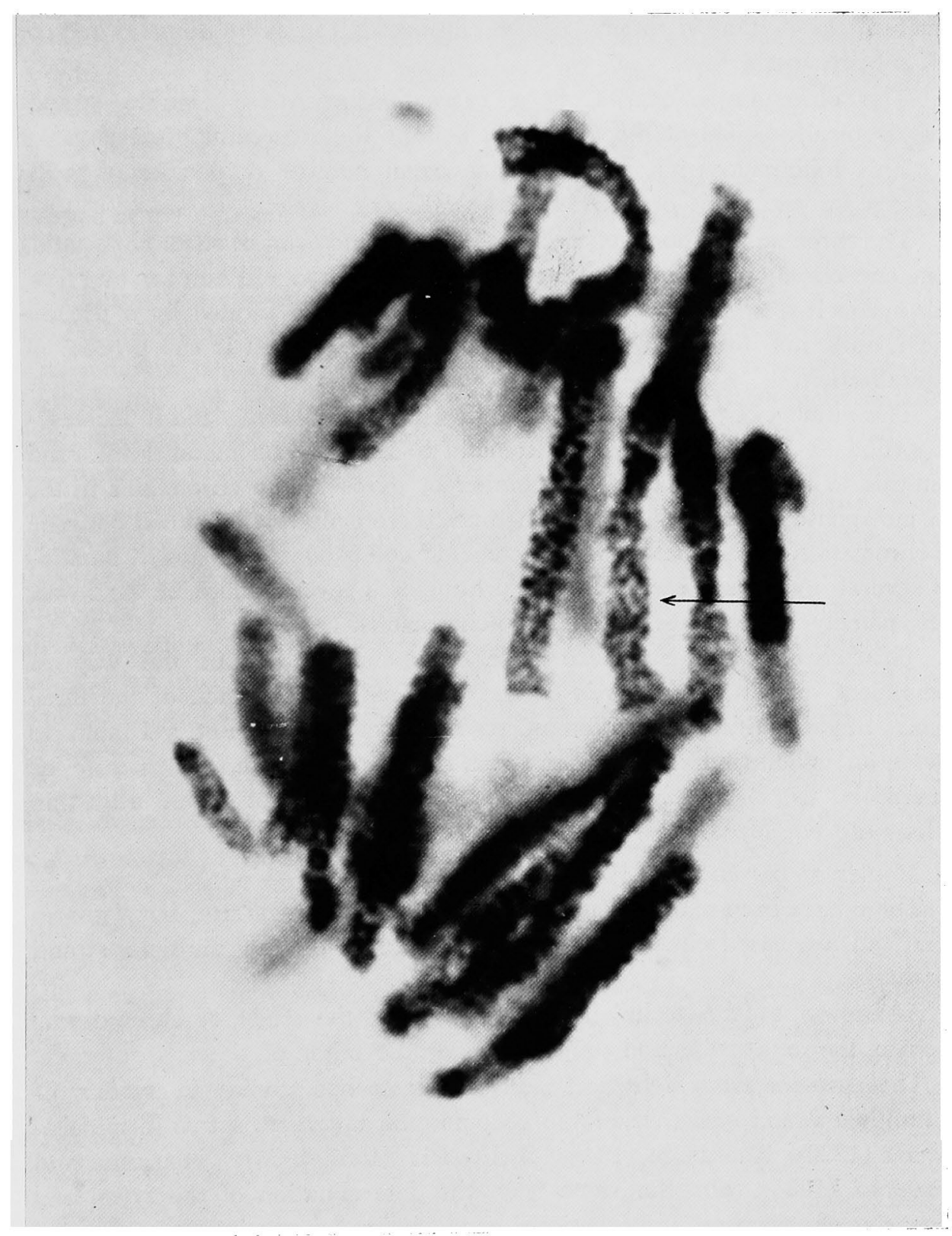

Fig. 3. Anaphase chromosomes showing the quatripartite structure and the plectonemic coil between half-sub-chromatids.

workers (reviewed by Kaufmann et al. 1960) have suggested that the chromosome is still further subdivided. Nebel $(1939,1941)$, Kuwada (1939) and Manton (1945) asserted that the anaphasic chromosome was made up of four 
strands. Our observations confirmed what was pointed out by these authors in relation to the fourfold structure of the anaphasic chromosome. Moreover, it has been possible for us to observe clearly the pattern of coiling at every structural level: that is to say, at the chromatid, the sub-chromatid and the half-sub-chromatid level.

The chromosome shows as a successive series of double strands plectonemically coiled at the different levels of the structural hierarchy.

This coiling does not presuppose an equal number or position of twists or gyres for each pair of strands at the different levels.

The chromatids of homologous chromosomes and even of sister chromatids from one chromosome, show differences in the position and number of gyres. This makes it reasonable to assume that the structure is formed by a process which does not follow a rigidly uniform course. Such is the process of despiralization.

The number of gyres is markedly different for each level, and it decreases proportionally from the half-sub-chromatid to the sub-chromatid level, and from this to the chromatid. The number of gyres in the chromatids in the late metaphase is very low, and sometimes there is no relational coil between the chromatids. Nevertheless, the plectonemic coil between the sub-chromatids has several gyres and each of these presupposes several more at the lower level, namely the level of the half-sub-chromatids.

This chromosome structure raises many difficulties in the way of observation, except when this is carried out separately at each of the three levels. Studies with the electron microscope are perhaps not yet able to provide an understanding of chromosome structure, because the structure, as observed by us, is bound to be very difficult to interpret when ultra-thin sections are employed.

\section{Models of chromosome structure}

There have been many theories about chromosome structure, but " grosso modo" all are based either on the single-strand or on the multiple strand hypothesis.

According to the techniques employed for the study of chromosome structure, the results obtained support one or the other of these hypotheses.

The evidence from light and electron microscope studies in favour of the multiple-strand structure of the chromosome at present seems formidable Kuwada (1939), Kaufmann (1948), Swift, Ris (1955-1956), Yasuzumi and Yamamoto (1953). In the same way, the determination of the types and frequencies of structural and functional modifications appearing in successive generations of the progeny of given individuals supports the multi-strand hypothesis (Review by Darlington (1955), Heitz (1953), Kaufmann $(1957,1960)$ ). On the other hand, autoradiographic studies, initiated by Taylor, support the theory of a single-strand anaphasic chromosome.

As a result of our observations, we have proof of a series of pairs of 
filaments at the different levels of the structural hierarchy. In a diploid cell (a common condition in higher organisms), each chromosome is represented twice, each chromosome is formed by two chromatids, each chromatid by two sub-chromatids, and each sub-chromatid by two half-sub-chromatids.

Therefore, the anaphasic chromosome is made up of four half-subchromatids, and the filaments invariably appear in pairs at each level.

To sum up: As a result of our observations it is logical to assume a multi-strand structure for the chromosome, and the pattern of coiling observed at the different levels supports the "rope" hypothesis, as expounded by Stefensen (1959) and Giménez-Martín et al.

\section{Theory of continuous despiralization}

The behaviour of chromosomes in the course of cell-division in relation to its different degrees of coiling and states of condensation is one of the most obscure and debated problems of cytology. Koller (1938) and Sparrow (1942) interpreted the mitotic condensation of the chromosome as a result of a spiralization process in the prophase (gyre initiation) and a despiralization process during the metaphase and anaphase (gyre elimination). In fact, these authors really applied to somatic chromosomes an interpretation of the facts demonstrated in the case of meiotic chromosomes, which have been more thoroughly investigated. As we have already noticed in the introduction, our observations and discussion relate exclusively to the structure and condensation of chromosomes during mitosis. A brief summary of the principal current belief with regard to this will indicate the present state of our knowledge on the subject:

1) In the prophase, chromosomes appear several times longer than metaphasic chromosomes. Then, a process of incipient coiling takes place within each chromatid, as the two chromatids disengage themselves by rotation of their end, eventually losing all the relational coils, whose origin goes back to the somatic coils left over from the anaphase of a previous mitosis.

2) In the metaphase, the chromosomes have already lost the last relational coils, and the chromatids come to lie side by side, with the functionally undivided centromere as the single fixed point of the chromosome. During metaphase, the decrease in the number of gyres proceeds, and is accompanied by an increase in their diameter. Consequently, there is a progressive condensation of the chromosome, its length decreases and its diameter increases. In the anaphase, the period of condensation continues.

3) In the late telophase the somatic coils (intrachromatidic spirals) begin to be eliminated, forming residual and relational coils. The interphase period has begun. According to Darlington (1955): "When the chromatids reconstitute daughter nuclei at the end of mitosis by absorbing water, they stretch these coils; but movement is limited by space and the coils fail to straighten. By a kind of hysteresis they are retained right through to the next mitosis, where they reappear as relic coils". As we have said when 
considering the structure of the chromosome under the light microscope, three levels can be observed: the chromatid, sub-chromatid and half-subchromatid level. Each of them is made up of two "units" of the lower level plectonemically coiled. The pattern of chromosome structure formed by this successive series of copies at the different levels makes it logical to assume that each level, in the following interphase, after a period of synthesis, becomes a higher level.

Therefore, there is a succession of levels, which in successive interphases become superior levels in the structural hierarchy of chromosomes. That is to say, the half-sub-chromatids, after a period of synthesis, become subchromatids change into chromatids, and the chromatids into chromosomes.

The decrease in the number of gyres from half-sub-chromatid to subchromatid, and the latter to chromatid, indicates a gyre elimination parallel to the step from one level to the level immediately above it during the cycle of cell division.

These observations indicate that the prophase spiralization of chromatids is unnecessary, for the prophase somatic coils may well be the result of the anaphase coils between the sub-chromatids, formed by the sub-chromatids coiled about each other. Thus the coils of the different levels might be the result of a continuous process of despiralization, which takes place as the counterpart of the reduplication of the structural units of the chromosome.

The spiralization could take place only during the interphase, in the period of synthesis of the elemental units, at the molecular level. This would be the first level of the structural hierarchy of the chromosome and the only one at which spiralization (formation of gyres) develops. According to this hypothesis, the origin of despiralization is the mechanical tensions developed within the chromosome by the duplication of the molecular strands. Under these circumstances, the different levels (plectonemically coiled) will tend to decrease the number of gyres, since the rigidity of the coiled system will be lessened as the diameter of the loops increases. The origin of the molecular spiralization would therefore be genetic, as well as the number of levels, but not the despiralization, which would give rise to mechanical tensions and would have only mechanical characteristics. This process of continued despiralization would take place throughout all division cycles and at all levels of the chromosome structure. The coils formed by intertwined chromatids and, in the same way, by sub-chromatids and half-sub-chromatids, would be a result of this continued despiralization, i.e. residual coils at different levels. According to our hypothesis the existence of external forces is not necessary to untwist the chromosome ends, nor are the hypothetical forces to induce the spiralization of chromatids in the prophase.

Continuous despiralization and chromosome condensation

In the light of this theory of continuous despiralization we can explain the condensation of the chromosome during mitosis. For this purpose we 
admit the following hypotheses:

1) The filament (elemental strand) constituting the structural basic unit presents no variations in its length during the cell division cycle. In mitosis this filament undergoes different changes in its coiling process but does not modify its length.

2) The spiralization levels causing the condensation of the chromosome lie beyond the decomposing range of the light microscope, i.e. they are submicroscopic levels. Thus, the coiling between sub-chromatids (somatic coils) is not the real cause of chromosome condensation.

With the aid of these working hypotheses, the continuous despiralization and the condensation of the chromosome can be explained as follows:

a) The mechanical tensions developing in the chromosome, as a result of the duplication of their structure, give rise to a continuous and progressive process of despiralization. As was mentioned, the duplication of each strand increases the tensions in the coil at the different levels while, on the other hand, the continued despiralization would give rise to a decrease in these tensions. The tensions arise from the molecular level, where the synthesis takes place, to the next level, and so on up to the chromatid level. In this way, we can explain the fact that the chromosomes untwist the chromatids, lying side by side in the late metaphase, and that the number of gyres is smaller between chromatids than between sub-chromatids, and in the latter smaller than between half-sub-chromatids.

At this moment, the gyres of the plectonemic coiling at each level would present a certain equilibrium between the coiling of strands and their rigidity. The new interphase, duplicating the structure at the different levels, induces an increase in the tensions of the gyres and, therefore, a new despiralization division of chromatids, which are duplicated sub-chromatids is initiated, until a new equilibrium is reached.

The chromosome condensation (enlargement and shortening of the chromosome) would be a result of the increase in gyre-diameter at all levels. By keeping the length of the structural basic unit, constant, the increase in gyre-diameter gives rise to a shortening and enlargement at this level. The progressive achievement of this result at all levels, reaching a maximum in the anaphase, would give rise to the remarkable condensation of the chromosome in these states. In the interphase, according to Darlington: "the chromatids reconstitute daughter nuclei by absorbing water, they stretch these coils", and so can facilitate a new synthesis.

b) On the other hand, to explain the condensation of the chromosome in mitosis we can also assume the existence of another mechanism, coming into action only during cell-division, together with the continuous despiralization, and overlapping this process.

This mechanism of chromosome condensation could give rise to the folding of the coils. In this way, the decrease in the number of gyres 
would be accompanied by a mutual approximation. This "packing" of the coils would increase the tension within the chromosome, thereby accentuating the process of despiralization still more during the metaphase and anaphase. In the telophase, during the formation of the membrane of the nucleus, the mechanism of condensation would stop and the spirals would relax, with the decrease in the rigidity of the chromosome, as it assumed its interphase structure. According to this hypothesis, the mechanism of condensation would begin to act at prophase and stop at telophase, i.e. it would manifest itself during the period when there is no nulear membrane. The coil "packing" would take place at a submicroscopic level. Nevertheless, the shortening thus induced must be observed at higher levels in the form of greater condensation and an increase in the tension of despiralization.

\section{References}

Abraham, A. 1939. Ann. Bot. Lond. N. S. 3: 545-68.

Darlington, C. D. 1955. Nature 176: 1139-1144.

Giménez-Martín et al. 1964. Cytologia (to be published).

Heitz, E. 1953. Arch. Julius Klaus-Stift. Vererbungsforsch. Sozialanthropol. u. Rassenhyg. 28: 260 .

Kaufmann, B. P. et al. 1960. International Review of Cytology 12: 77-128.

- 1948. Bot. Rev. 14: 57-126.

Koller, P. C. 1938. Jour. Genetics 36: 177-195.

Kuwada, Y. 1939. Cytologia 10: 213-256.

Manton, I. 1939. Phil. Trans. Royal Soc. London, B. 230: 179-216.

- 1945. Amer. Jour. Bot. 32: 342-348.

Nebel, B. R. 1941. Cold Spring Harbor Symp. Quant. Biol. 9: 7-12.

- 1939. Bot. Rev. 5: 563-626.

Ris, H. 1955. Fine structure of cells. Symposium held at the 8th Congress of Cell Biology p. 121, P. Noordhoff Ltd., Groningen The Netherlands.

- 1957. A Symposium on the Chemical Basis of Heredity, p. 23 W. D. McElroy and B. Glass, Editors, The Johns Hopkins Press, Baltimore.

Sparrow, A. H. et al. 1941. Can. Jour. Res. 19: 323-350.

- 1942. Can. Jour. Res., D. 20: 257-266.

Steffesen, D. 1959. Brookhaven Symposia in Biology. N. 12: 103-124.

Swift, H. Molecular Control of Cell Activity p. 73 Ed. J. M. Allen, Mograw-Hill-Book Co. N. Y. Taylor, J. H. 1958. Genetics 43: 515.

Tjio, J.H. and Levan, A. 1950. Ann. Estac. Exptl. Aula Dei 2: 21.

Yasuzumi, G. and Yamamoto, Y. 1953. Cytologia 18: 240. 